Conclusion Treatment with lubiprostone resulted in increased SBM frequencies and improvement in related symptoms in patients with chronic idiopathic constipation regardless of age, gender, or race.

Disclosure of Interest None Declared.

\section{PTH-197 LONG-TERM EFFICACY OF LUBIPROSTONE DEMONSTRATED IN PATIENTS WITH CONSTIPATION REGARDLESS OF AGE, GENDER OR RACE}

doi:10.1136/gutjnl-2013-304907.684

1."T Joswick, 'G Dolecek, ${ }^{2} \mathrm{P}$ Lichtlen, ${ }^{3} \mathrm{D}$ Panigrahi, ${ }^{4} \mathrm{R}$ Ueno. ${ }^{1} R \& D$, Sucampo Pharmaceuticals, Bethesda, United States; ${ }^{2} R q D$, Sucampo Pharmaceuticals, Zug, Switzerland; ${ }^{3}$ Medical Affairs, Sucampo Pharmaceuticals, Bethesda, United States; ${ }^{4}$ Sucampo Pharmaceuticals, Zug, Switzerland

Introduction Constipation affects millions globally and may require chronic treatment. In 24- and 48-week trials, lubiprostone demonstrated safety and efficacy in long-term treatment of adults with chronic idiopathic constipation. Analyses of lubiprostone's (24 mcg BID) efficacy in age, gender and race subgroups were performed.

Aims and Methods Pooled data from three Phase 3 open-label safety studies were reviewed to analyse efficacy in the following subpopulations: non-elderly ( $<65$ years) and elderly ( $\geq 65$ years); male and female; and non-white and white. Constipation severity, abdominal bloating, and abdominal discomfort were rated on a 5 -point scale ranging from absent $=0$, mild $=1$, moderate $=2$, severe $=3$ and very severe $=4$, and changes from baseline following treatment with lubiprostone were analysed.

Results In the non-elderly and elderly, lubiprostone improved constipation severity $(p \leq 0.0001)$ each week beginning at Week 1 and continuing through Week 48. Among males and females, lubiprostone improved ( $p \leq 0.0001)$ constipation severity each week with similar findings $(p \leq 0.0001)$ for non-whites and whites, For abdominal discomfort, significant changes were seen at all weeks in the non-elderly $(p \leq 0.0001)$ and for all weeks in the elderly $(p \leq 0.0001$ to $p=0.0150)$ except Week $8(p=0.0530)$. Among the genders, improvements occurred at all weeks in males $(p \leq 0.0001$ to $p=0.0220)$ and in females $(p \leq 0.0001)$. By race, improvements occurred at all weeks among nonwhites $(p \leq 0.0001$ to $p=0.0020)$ and among whites $(p \leq 0.0001)$. Significant changes in abdominal bloating were seen in the non-elderly $(p \leq 0.0001)$ and for the elderly $(p \leq 0.0001$ to $p=0.0180)$ at all weeks. Abdominal bloating improved at all weeks for males ( $p \leq 0.0001$ to $p=0.0010)$ and females $(p \leq 0.0001)$. For non-whites and whites, abdominal bloating improved $(p \leq 0.0001)$ at all weeks.

\section{Abstract PTH-197 Table 1 Change from Baseline for Constipation Severity}

\begin{tabular}{llcccccc}
\hline & & \multicolumn{6}{c}{ Mean Change From Baseline } \\
\hline \multirow{3}{*}{ Age } & & Baseline & Week 1 & Week 12 & Week 24 & Week 36 & Week 48 \\
& $<65$ Years & 2.91 & -1.21 & -1.18 & -1.21 & -1.19 & -1.14 \\
\multirow{3}{*}{ Gender } & $\geq 65$ years & 2.76 & -1.11 & -0.97 & -1.00 & -1.15 & -1.03 \\
& Male & 2.65 & -1.21 & -1.12 & -1.18 & -1.27 & -1.17 \\
& Female & 2.92 & -1.19 & -1.14 & -1.17 & -1.16 & -1.11 \\
Race & Non-Whites & 2.98 & -1.52 & -1.51 & -1.52 & -1.48 & -1.38 \\
& Whites & 2.87 & -1.14 & -1.08 & -1.12 & -1.13 & -1.08 \\
\hline
\end{tabular}

Conclusion Lubiprostone demonstrated long-term efficacy through an overall improvement in constipation severity for up to 12 months regardless of age, gender, or race. Similarly, improvements were noted in abdominal bloating and abdominal discomfort for both race and gender subpopulations along with some significant and positive trend for improvement in the age group analyses. Disclosure of Interest None Declared.

\section{PTH-198 POOLED ANALYSIS OF THE MOST FREQUENT ADVERSE EVENTS ASSOCIATED WITH THE USE OF LUBIPROSTONE}

doi:10.1136/gutjnl-2013-304907.685

1. ${ }^{*} \mathrm{~T}$ Joswick, ${ }^{1} \mathrm{G}$ Dolecek, ${ }^{2 P}$ Lichtlen, ${ }^{3} \mathrm{D}$ Panigrahi, ${ }^{4} \mathrm{R}$ Ueno. ${ }^{1} R \notin D$, Sucampo Pharmaceuticals, Bethesda, United States; ${ }^{2} R \& D$, Sucampo Pharmaceuticals, Zug, Switzerland; ${ }^{3}$ Medical Affairs, Sucampo Pharmaceuticals, Bethesda, United States, ${ }^{4}$ Sucampo Pharmaceuticals, Zug, Switzerland

Introduction Lubiprostone, a novel $\mathrm{ClC}-2$ activator, has been shown to be efficacious and well-tolerated by patients with chronic constipation in short- and long-term clinical trials. To better characterise the most frequent adverse events (AEs) associated with the use of lubiprostone $24 \mathrm{mcg} \mathrm{BID}$, we examined pooled results of patients enrolled in Phase 2 and 3 trials of 3 to 48 weeks' duration. Methods Data for all safety-evaluable patients were pooled and compared between treatment groups (placebo vs lubiprostone 24 mcg BID). Nausea, headache, and diarrhoea AEs were analysed in terms of severity, duration, frequency, action taken (eg, drug withdrawn, dose reduced), and outcome. In addition, nausea-related variables were explored in subpopulations of elderly ( $\geq 65$ years of age) and male patients.

Results The pooled population included 1113 lubiprostone and 316 placebo patients. Approximately 16\% were elderly and 13\% were male. At least $1 \mathrm{AE}$ occurred in $79.1 \%$ of lubiprostone patients, which included $31.1 \%$ with nausea, $13.2 \%$ with headache, and $13.2 \%$ with diarrhoea. At least $1 \mathrm{AE}$ occurred in $39.6 \%$ of placebo patients, which included $5.1 \%$ with nausea, $6.6 \%$ with headache, and $0.9 \%$ with diarrhoea. Of those lubiprostone patients who experienced nausea, $88.7 \%$ reported nausea to be mild or moderate in severity. Overall, $74.5 \%$ of nausea events reported by lubiprostone patients were intermittent, and the median durations of nausea events were 12 and 7.5 days for lubiprostone and placebo patients, respectively. Notably, mean incidences of nausea per patient were 1.3 and 1.0 for lubiprostone and placebo patients, respectively. With regard to action taken, $64.1 \%$ of nausea events required no change in treatment regimen, and $9.6 \%$ of events resulted in dose reduction. Nausea was less prevalent in subpopulations, occurring in only $18.8 \%$ and $8.2 \%$ of lubiprostone elderly and male patients, respectively, compared with $34.5 \%$ of female patients. Similarly, the majority of lubiprostone patients experiencing headache and diarrhoea had mild-to-moderate symptom severity $(89.8 \%$ and $82.3 \%$, respectively). Furthermore, headache and diarrhoea events in lubiprostone patients were mostly intermittent (64.2\% and $74.0 \%$, respectively), and the median duration of these events were 9 and 4 days, respectively. Discontinuation rates due to nausea, headache, and diarrhoea were $8.7 \%, 3.7 \%$, and $2.2 \%$, respectively.

Conclusion Nausea, headache, and diarrhoea associated with lubiprostone use are generally mild to moderate in severity, intermittent, and limited in duration.

Disclosure of Interest None Declared.

\section{PTH-199 PREVALENCE OF ORGANIC DISORDERS IN CONSECUTIVE NEW PATIENTS MEETING CRITERIA FOR IBS IN A GASTROENTEROLOGY CLINIC}

doi:10.1136/gutjnl-2013-304907.686

1.*U Shivaji, 'D Gracie, 'A Sainsbury, 'A C Ford. 'Gastroenterology, Leeds Teaching Hospitals, Leeds, UK

Introduction A positive diagnosis of irritable bowel syndrome (IBS), without the need for recourse to investigation, is encouraged. Patients meeting symptom-based diagnostic criteria for IBS are often given reassurance that there is no serious underlying pathology, and treated symptomatically. However, some studies have suggested that an organic diagnosis, such as coeliac disease or pancreatic 